\title{
Improved implementation and robustness study of the X-FEM for stress analysis around cracks
}

\author{
E. Béchet ${ }^{1}$, H. Minnebo ${ }^{1}$, N. Moës ${ }^{1, *, \dagger}$ and B. Burgardt ${ }^{2}$ \\ ${ }^{1}$ Institut de Recherche en Génie Civil et Mécanique, UMR CNRS 6183, Ecole Centrale de Nantes, \\ B.P. 92101, 44321 Nantes Cedex 3, France \\ ${ }^{2}$ SNECMA Moteurs, Rond Point René Ravaud-Réau, 77550 Moissy-Cramayel, France
}

\begin{abstract}
Numerical crack propagation schemes were augmented in an elegant manner by the X-FEM method. The use of special tip enrichment functions, as well as a discontinuous function along the sides of the crack allows one to do a complete crack analysis virtually without modifying the underlying mesh, which is of industrial interest, especially when a numerical model for crack propagation is desired. This paper improves the implementation of the X-FEM method for stress analysis around cracks in three ways. First, the enrichment strategy is revisited. The conventional approach uses a 'topological' enrichment (only the elements touching the front are enriched). We suggest a 'geometrical' enrichment in which a given domain size is enriched. The improvements obtained with this enrichment are discussed. Second, the conditioning of the X-FEM both for topological and geometrical enrichments is studied. A preconditioner is introduced so that 'off the shelf' iterative solver packages can be used and perform as well on X-FEM matrices as on standard FEM matrices. The preconditioner uses a local (nodal) Cholesky based decomposition. Third, the numerical integration scheme to build the X-FEM stiffness matrix is dramatically improved for tip enrichment functions by the use of an ad hoc integration scheme. A $2 \mathrm{D}$ benchmark problem is designed to show the improvements and the robustness.
\end{abstract}

KEY WORDS: X-FEM; convergence rate; integration scheme; preconditioner; $J$-integral; crack propagation

\section{INTRODUCTION}

Typical finite element analysis for crack propagation involves three major steps. The first step involves the construction of a mesh that matches exactly the crack geometry. This is often

\footnotetext{
*Correspondence to: Nicolas Moës, Institut de Recherche en Génie Civil et Mécanique, Ecole Centrale de Nantes, B.P. 92101, 1 rue de la Noë, 44321 Nantes Cedex 3, France.

†E-mail: nicolas.moes@ec-nantes.fr

Contract/grant sponsor: SNECMA Moteurs
} 

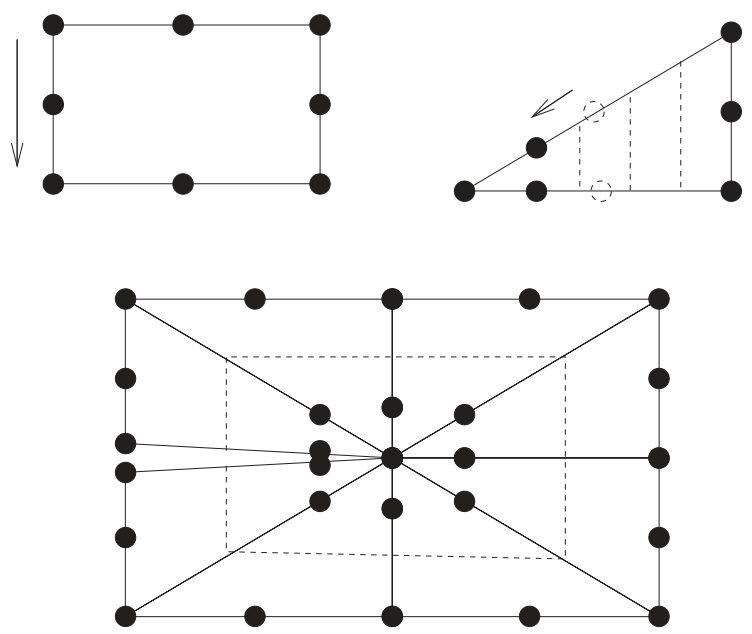

Figure 1. Barsoum elements around a crack tip in 2D.

cumbersome, because the geometry of the crack is independent of the CAD model used to describe the computational domain. Moreover, when the crack evolves, the mesh should evolve and must conform the crack geometry. The second step involves the computation of stress intensity factors. These are used for instance to predict the path followed by the crack if fatigue behaviour is considered. The computation of stress intensity factors must be carefully done. In this matter, domain integrals are found to be more accurate than extrapolations made at the crack tip. The integration domain must be sufficiently large so as to avoid numerical inaccuracies. To further lower these inaccuracies and possibly reduce the size of the domain integrals, special crack tip elements can be used. Among those, Barsoum [1] elements are a good choice if one wants to keep the standard finite elements approach. Barsoum elements rely on standard quadratic elements, in which specific control points are moved to the quarter of the edges, in addition to the collapse of one of its faces (see Figure 1). There are few, if any, modifications of the finite element codes dealing with Barsoum elements. The third and last step in crack path prediction involves the update of the crack front. As the crack is part of the mesh, this involves the update of the mesh, as well as the data structures associated with the crack geometry. This geometrical update is an issue with complex models coming from industry. The X-FEM method can handle this by means of the partition of unity $[2,3]$. This method can handle discontinuities either in the primary field (displacement) or in the derivatives independently of the mesh by explicitly constructing an appropriate approximation space. The X-FEM uses the partition of unity property [2] to define special enrichment functions that represent the discontinuity across the crack. These functions are (1) a modified Heaviside function to represent the jump across the crack [4], and (2) the Westergaard asymptotic near tip displacement field [5]. The regular shape functions are multiplied by those functions to form the enriched shape functions. The partition of unity states that the new function space, which is the combination of regular and enriched shape functions, is able to represent the crack kinematics, totally eliminating re-meshing. Basically, this enrichment scheme allows for the same order of convergence of regular finite elements (with or without Barsoum elements). 
The convergence properties of these methods are low, because of the crack tip singularity in the stress field. However, the enrichment in the X-FEM is very versatile. For instance, it is possible to let arbitrary functions appear in the function space, and the enrichment domain can be chosen as wide as necessary. While many studies have been done with the Westergaard enrichment made only in the elements immediately around the crack tip, few have examined the convergence properties if the enrichment domain is larger. Beside this, there are issues in the X-FEM implementation that deal with the nature of the enrichment. In Barsoum elements, integration is easy because the modified mapping in the tip elements (that brings asymptotic shape functions), is also used for the integration. In this case, the integration is exact for elastic problems. In the X-FEM, there is no 'out of the box' rule to integrate the shape functions as they are defined by the user. We must use a standard Gauss rule, or provide a quadrature of our own. Strouboulis et al. [6] proposed to use the DCUHRE algorithm [7] for this purpose. This is however restricted to quadrilaterals. We propose another scheme in this paper, that is adapted to unstructured triangular meshes. Beside this, the conditioning of linear systems obtained from the X-FEM can be very bad under some circumstances, and especially in fracture mechanics because of the type of the enrichment functions involved. While this might not be an issue with direct solvers, the method must also be attractive for iterative solvers. The convergence properties of iterative solvers are closely related to the structure of the linear system, and the use of a specific (application dependent) preconditioner is often necessary. The X-FEM does not infringe this rule of thumb, and a specific preconditioner is proposed. To be more precise, we introduce a preconditioning operator after which a standard preconditioner for the FEM performs well.

\section{MECHANICAL MODEL}

The equations of elastostatics under small strains and small displacements are recalled here. The cracked domain is denoted by $\Omega$ and its boundary $\partial \Omega \cup C^{+} \cup C^{-}$by $B$. The boundary $B$ is subjected to boundary conditions: $B_{t}$ where tractions are imposed (natural boundary condition), $B_{u}$ where the displacement $\mathbf{u}$ is imposed (essential boundary condition), and the crack faces $C^{+}$and $C^{-}$, both assumed to be traction-free. The Cauchy stress tensor $\sigma$ must then satisfy the equilibrium equations

$$
\begin{aligned}
& \nabla \cdot \boldsymbol{\sigma}=\mathbf{0} \quad \text { on } \Omega, \quad \boldsymbol{\sigma} \cdot \mathbf{n}=\mathbf{t} \quad \text { on } B_{t} \\
& \boldsymbol{\sigma} \cdot \mathbf{n}=\mathbf{0} \quad \text { on } C^{+}, \quad \boldsymbol{\sigma} \cdot \mathbf{n}=\mathbf{0} \quad \text { on } C^{-}
\end{aligned}
$$

The strain-displacement relationship and constitutive law are

$$
\begin{aligned}
& \varepsilon=\frac{1}{2}\left(\nabla \mathbf{u}+(\nabla \mathbf{u})^{\mathrm{T}}\right) \quad \text { in } \Omega, \quad \mathbf{u}=\mathbf{u}_{d} \quad \text { on } B_{u} \\
& \boldsymbol{\sigma}=\mathbf{C}: \varepsilon \quad \text { in } \Omega
\end{aligned}
$$

where $\mathbf{C}$ is Hooke's elasticity tensor. 


\section{STRESS INTENSITY FACTORS COMPUTATION}

The evolution of the crack depends on stress intensity factors. These are computed using well known domain integrals initiated by Destuynder [8], as well as the more widely available paper from Shih [9]. These are easier to implement in the finite element method than their contour integral counterpart. The transformation is done using Gauss's theorem and a virtual velocity function. We recall briefly the expression of the density of energy release from the Eshelby energy-momentum tensor $\mathbf{P}$ for a plane crack (Figure 2). The contour $\Gamma$ lies on the boundary of the domain $S$. In the sequel, $\mathbf{n}_{j}^{\{c \mid \Gamma\}}$ is the normal to the crack faces and the contour, respectively. The virtual velocity of the crack at the considered location is $\mathbf{v}_{m}$. The contour is displayed in Figure 3

$$
\begin{gathered}
\mathbf{P}_{m j}=\frac{1}{2} \boldsymbol{\sigma}_{k l} \boldsymbol{\varepsilon}_{k l} \delta_{m j}-\boldsymbol{\sigma}_{i j} \mathbf{u}_{i, m} \\
J=\oint_{\Gamma} \mathbf{v}_{m} \mathbf{P}_{m j} \mathbf{n}_{j}^{\Gamma} \mathrm{d} s
\end{gathered}
$$

The expression (6) [10] can be expanded into a domain integral with help of Gauss's theorem

$$
J=-\int_{S} \mathbf{q}_{m, j} \mathbf{P}_{m j} \mathrm{~d} S+\int_{C^{+} \cup C^{-}} \mathbf{q}_{m} \mathbf{P}_{m j} \mathbf{n}_{j}^{c} \mathrm{~d} s
$$

where

$$
\mathbf{q}_{m}=\alpha \mathbf{v}_{m}
$$

In this expression, $\alpha$ is a weight factor vanishing on the domain boundary and is 1 at the crack tip. If there are no forces acting on crack sides $C^{+}$and $C^{-}$and if the virtual velocity $\mathbf{v}_{m}$ is in the crack plane, the second part of the integral vanishes even if the crack path is curved. We use the same idea to compute interaction integrals [11]

$$
I^{a}=-\int_{S} \mathbf{q}_{m, j} \mathbf{P}_{m j}^{a} \mathrm{~d} S-\int_{S} \mathbf{q}_{m j} \mathbf{P}_{m, j}^{a} \mathrm{~d} S+\int_{C^{+} \cup C^{-}} \mathbf{q}_{m} \mathbf{P}_{m j}^{a} \mathbf{n}_{j}^{c} \mathrm{~d} s
$$

The Eshelby-like tensor $\mathbf{P}^{a}$ is defined as the following (see Reference [12]), because of the main symmetry of the elasticity tensor $\mathbf{C}_{i j k l}=\mathbf{C}_{k l i j}$

$$
\mathbf{P}_{m j}^{a}=\boldsymbol{\sigma}_{k l} \boldsymbol{\varepsilon}_{k l}^{a} \delta_{m j}-\boldsymbol{\sigma}_{i j}^{a} \mathbf{u}_{i, m}-\boldsymbol{\sigma}_{i j} \mathbf{u}_{i, m}^{a}
$$

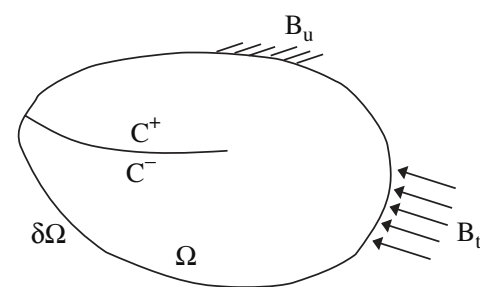

Figure 2. Cracked body with boundary conditions. 


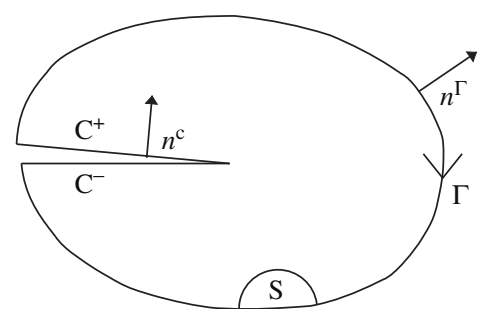

Figure 3. Contour integrals path.

In Equation (10), $\boldsymbol{\sigma}^{a}, \varepsilon^{a}, \mathbf{u}^{a}$ are the auxiliary fields for which every $K_{i}^{a}$ is known. Using three linearly independent sets of auxiliary fields $A^{a}=\left\{\boldsymbol{\sigma}^{a}, \varepsilon^{a}, \mathbf{u}^{a}\right\}$, each of those corresponding to $K_{i}^{a}=\delta_{i a}$, one can eventually determine the values of the stress intensity factors for the actual displacement field with the relation between the SIFs and the energy release rate $G$

$$
G^{a}=2 \frac{1-v^{2}}{E}\left(K_{1} K_{1}^{a}+K_{2} K_{2}^{a}\right)+\frac{1}{\mu} K_{3} K_{3}^{a}
$$

and

$$
G^{a}=\frac{I^{a}}{\left.\alpha\right|_{\text {tip }}}=I^{a}
$$

The choice of the scalar $\alpha$ defines the actual domain $S$ on which the SIF calculations take place. In the sequel, $\alpha$ will always be chosen as a linear field

$$
\alpha=\sum_{i} N_{i} \alpha_{i}
$$

In this relation, $N_{i}$ is the linear Lagrange shape function based on node $n_{i}$. For every node of the triangulation $T^{h}$ of the domain $\Omega$, the following relation holds:

$$
\alpha_{i}= \begin{cases}1 & \text { if } \operatorname{support}\left(N_{i}\right) \subset S \\ 0 & \text { otherwise }\end{cases}
$$

This means that the domain $\Gamma$ in the integrals (6) and (7) is approximated by the elements of the set $S_{\Gamma}$, which are all the elements of $S$ touching the boundary of the domain, where $\alpha$ is vanishing. The resulting SIF and energy release rate computed via domain integrals are in fact a weighted mean of the same variables computed with contour integrals, on contours passing inside the set $S_{\Gamma}$.

\section{UPDATED X-FEM FORMULATION}

Partition of unity methods were introduced by Babuška and Melenk [2]. The idea is to add user defined functions in the regular finite element spaces. In fracture mechanics, this is desirable in order to represent the discontinuity of the displacement field across the fracture surface, and incorporate the specific asymptotic displacement field at the crack tip. 


\subsection{Enrichment functions}

The X-FEM method, based on the local PUM [4,5,11], uses elementary displacement fields obtained from an infinite cracked body. There are 4 basic enrichment functions needed to span the Westergaard solution at the crack tip $[5,13]$

$$
g_{i}(r, \theta)=\left\{\sqrt{r} \sin \frac{\theta}{2}, \sqrt{r} \cos \frac{\theta}{2}, \sqrt{r} \sin \frac{\theta}{2} \sin \theta, \sqrt{r} \cos \frac{\theta}{2} \sin \theta\right\}
$$

In these enrichment functions, $r$ and $\theta$ are polar co-ordinates respective to the crack front. Only elements immediately around the crack tip are usually enriched with these functions (in the sequel, this will not be necessarily the case). If one examines the Westergaard tip functions, the first term $g_{1}$ is able to represent a discontinuity in the displacement field. For the remainder of the domain, we have to provide a discontinuous function of our own. So, along the sides of the crack the enrichment is chosen to be the modified Heaviside discontinuous function so that the resulting displacement field contains a discontinuity at the location of the crack [4]

$$
h(\varphi)= \begin{cases}+1 & \text { if } \varphi \geqslant 0 \\ -1 & \text { if } \varphi<0\end{cases}
$$

In this function, $\varphi$ is the signed distance computed normally to the crack sides. In the remaining portion of the domain, there is no enrichment so the conventional finite element basis is the only one existing. The resulting displacement field is

$$
\mathbf{u}^{h}=\sum_{i \in R} \mathbf{N}_{i} \alpha_{i}+\sum_{i \in T} \sum_{j=1 \ldots 4} \mathbf{N}_{i} g_{j} \beta_{i j}+\sum_{i \in H} \mathbf{N}_{i} h \gamma_{i}
$$

Here $R, T$ and $H$ are, respectively, the regular set of nodes having the regular finite element basis, the set of nodes which need to be enriched for the tip functions, and the set of nodes for the Heaviside enrichment. The set of nodes $H$ is determined to avoid a linear dependency, as in Reference [4]. The coefficients $\alpha_{i}, \beta_{i j}$ and $\gamma_{i}$ are the degrees of freedom of the displacement field, determined by solving the linear system obtained after assembly. The integration in standard finite element codes is generally done with a Gauss quadrature. The result of this quadrature is very accurate if one uses a sufficient number of Gauss points, because the terms to integrate are generally polynomials. In the X-FEM, since the enrichment functions are not polynomials, integration is not expected to be exact with a Gauss quadrature rule. This issue will be discussed in the sequel.

\subsection{New enrichment procedures}

As said previously, the enrichment in the X-FEM (also with the Barsoum element) is such that only one layer of elements bears the complete enrichment basis. One drawback of this enrichment scheme is that the accuracy depends also on the position of the crack tip (for instance close to a node or an edge). In addition, Chessa et al. [14] has shown under certain circumstances the poor accuracy of computations because a layer of elements are only partially enriched. This enrichment scheme is referred later in the discussion as 'topological'. The characteristic of this 'topological' behaviour is that the size of the enriched area is proportional to $h$. By contrast, we propose a 'geometrical' enrichment, that bears the characteristic of

a constant enriched area within a prescribed geometry. In fact, in Reference [3, p. 23], an 


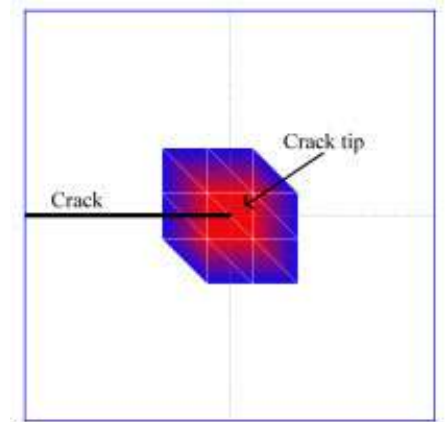

(a)

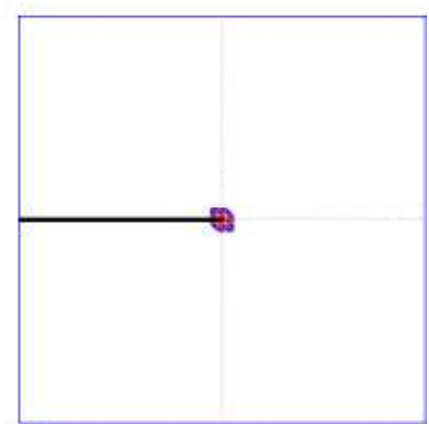

(b)

Figure 4. Selected elements for the topological enrichment: only the elements touching the crack tip are enriched. The enriched area therefore depends on the mesh density: (a) $h=\frac{1}{10}$; and (b) $h=\frac{1}{50}$.

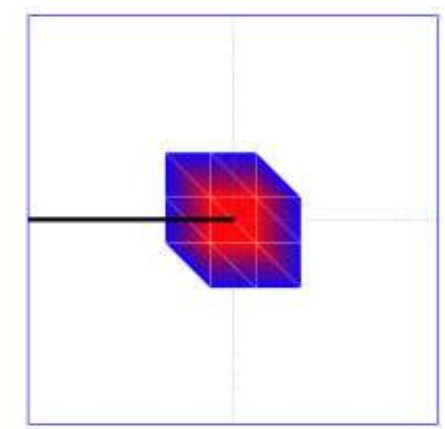

(a)

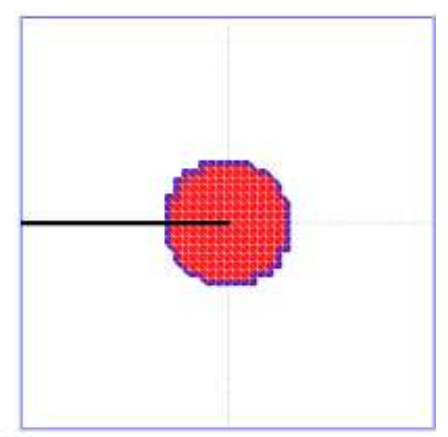

(b)

Figure 5. Selected elements for the geometrical enrichment: a zone of a given size is enriched regardless of the mesh density: (a) $h=\frac{1}{10}$; and (b) $h=\frac{1}{50}$.

implicit reference to additional layers of elements in the enriched area is briefly made. In the Figures 4 and 5, a comparison showing enriched areas for both methods with two levels of refinement is displayed. The criteria used to determine which nodes are enriched is such that if one node lies within a circle of radius $r_{e}$, then it is enriched.

\subsection{Integration issue}

With the X-FEM enrichment functions, singular integrals must be computed accurately. The four crack tip functions are of the following type:

$$
g(r, \theta)=\sqrt{r} f(\theta)
$$

where $f$ is the product of harmonic functions. The singularity occurs in the integrand of related terms, in the stiffness matrix. Those terms are the product of the gradient of two interpolation 


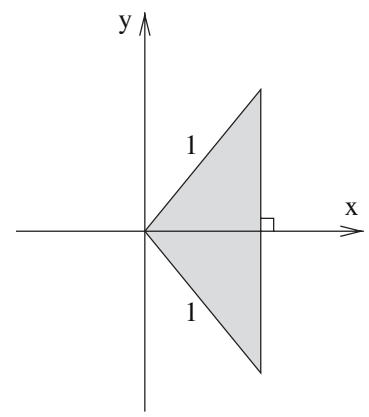

Figure 6. Element used to integrate various singular functions.

functions, possibly enriched. By substitution, we can easily find that the functions appearing in the integrand are a combination of $\{1 / r, 1 / \sqrt{r}, 1, \sqrt{r}, r\}$ multiplied by harmonic functions, in addition to terms coming from regular shape functions. The harmonic functions are not singular, only the radial parts create difficulties for the integration. In the sequel, we focus on the integration of $g(r, \theta)$ where $g$ is harmonic in $\theta$ and singular in $r$.

Previously, triangular sub-elements were created in order to have continuous functions on every sub-element. The singular point therefore lies on a vertex. As shown in Figure 7, the convergence achieved with a standard Gauss integration is very slow. The need for a better quadrature scheme is clear. This convergence curve has been generated by integrating on the element shown in Figure 6.

Another option available to integrate crack singularities is the use of the so-called 'quarterpoint' Barsoum element described earlier in the text [1]. The drawbacks of this method are (a) the mesh have to conform to the crack's, geometry, and (b) Barsoum elements do not hold a radial parameter $r$, so we would only be able to represent functions of $\sqrt{u}, u$ being a linear (not radial) parameter whose value is zero at the singular point and one along the outer edge of the triangle. In addition, in $3 \mathrm{D}$, this quadrature is valid only on the edges of the singular elements (Figure 1).

\subsection{Description of the singular mapping}

The purpose is to integrate, in a fast way and within a prescribed accuracy, the function $g(r, \theta)$ on a triangular patch. For this, we will map the real triangular interpolation cell on a reference quadrangular cell. This way we transform singular functions into regular ones. The mapping is shown in Figure 8, with Node 0 (singular one) corresponding to the reference edge $u=-1$.

On the reference element, $u$ represents the radial co-ordinate, and $v$ represents the orthoradial one. These co-ordinates are obtained after several co-ordinate changes starting from a polar transformation $(r, \theta)$. In the sequel, we want to integrate the expression

$$
I=\iint_{T} g(x, y) \mathrm{d} S
$$

We introduce the polar co-ordinates $x(r, \theta)=r \cos \theta$ and $y(r, \theta)=r \sin \theta$. In the following expression, $r_{0}$ is the orthogonal distance of the opposite side to the singular node; it also gives 


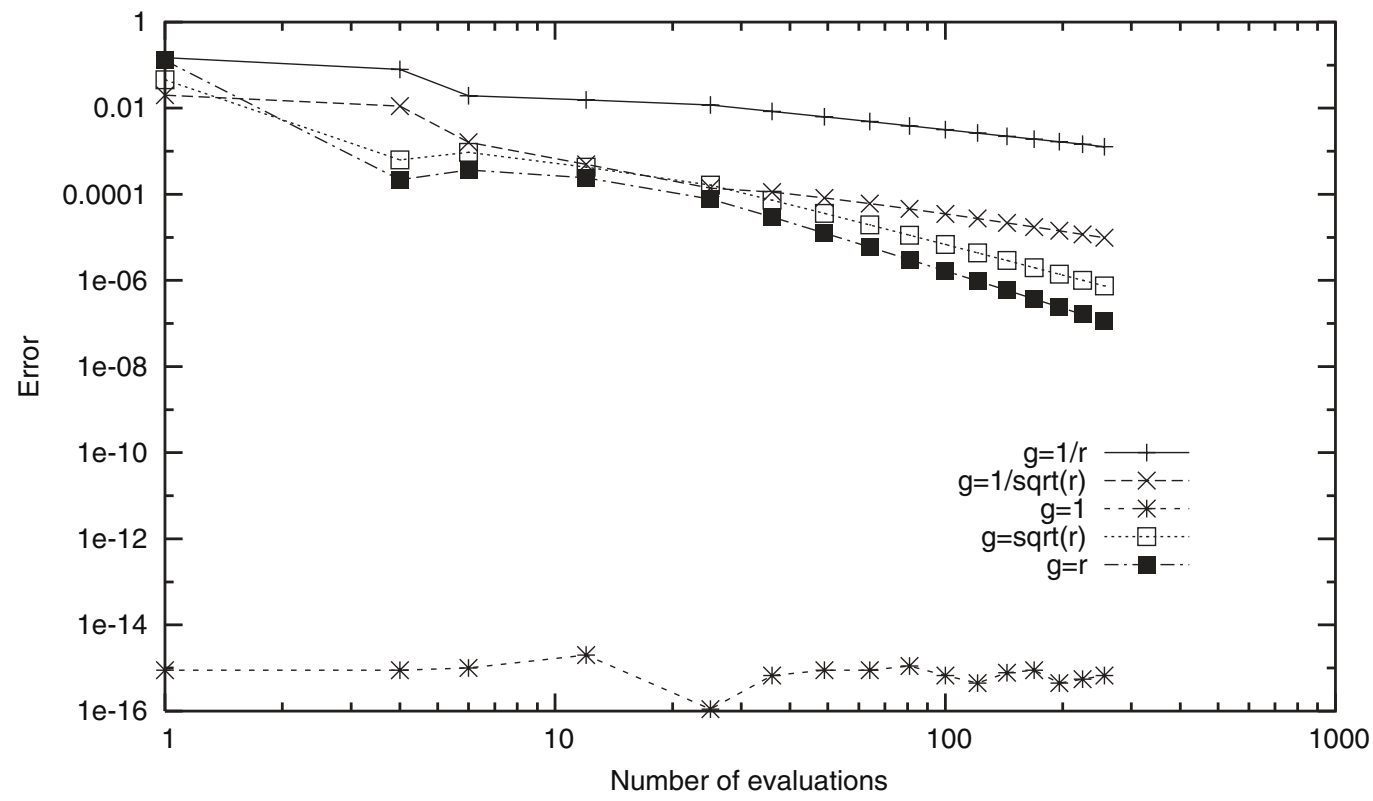

Figure 7. Convergence of a standard Gauss-Legendre integration scheme.

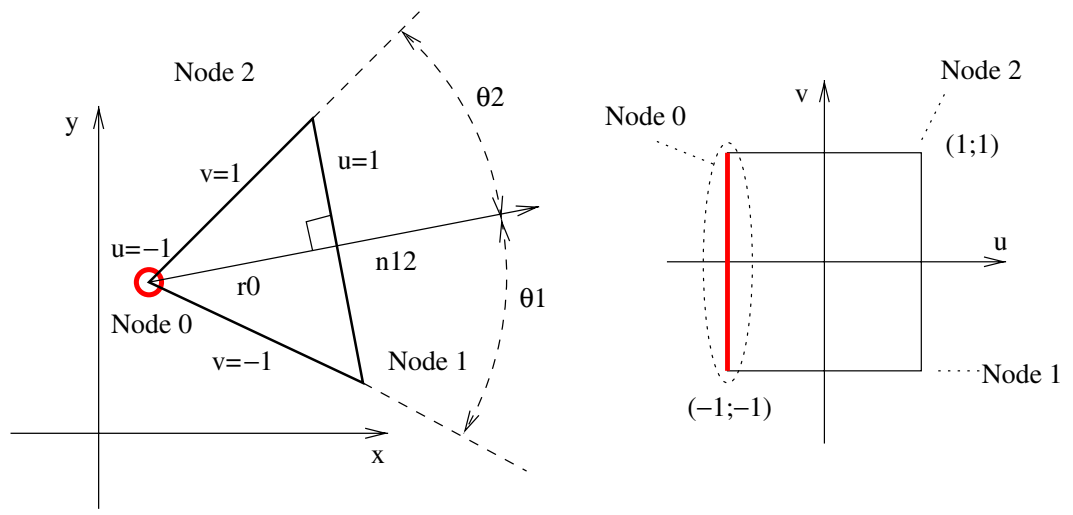

Figure 8. Triangular element and quadrangular reference element.

the reference angle for $\theta$. We now have

$$
I=\iint_{T} g(x(r, \theta), y(r, \theta)) r \mathrm{~d} r \mathrm{~d} \theta \quad \text { with } r \in\left[0 ; \frac{r_{0}}{\cos \theta}\right], \quad \theta \in\left[\theta_{1}, \theta_{2}\right]
$$

We then use the following mapping in order to fit $u$ in the interval $[-1 ; 1]$ and have polynomial terms with respect to $\sqrt{r}$

$$
r=r_{0} \frac{(1+u)^{2}}{4 \cos \theta}, \quad \mathrm{d} r=r_{0} \frac{1+u}{2 \cos \theta} \mathrm{d} u
$$


Substituting into expression (20) yields

$$
\begin{aligned}
& I=\iint_{T} r_{0} \frac{1+u}{2 \cos \theta} g(x(r(u, \theta), \theta), y(r(u, \theta), \theta)) r(u, \theta) \mathrm{d} u \mathrm{~d} \theta \\
& \quad \text { with } u \in[-1 ; 1], \quad \theta \in\left[\theta_{1}, \theta_{2}\right]
\end{aligned}
$$

In this expression, a term in $(\cos \theta)^{-1}$ appears, which can be singular. We introduce then another co-ordinate change $t$ so that $\mathrm{d} t=(\cos \theta)^{-1} \mathrm{~d} \theta$ as proposed in Reference [15]. It brings

$$
t=\frac{1}{2} \ln \left(\frac{1+\sin \theta}{1-\sin \theta}\right) \quad \text { or } \quad \theta=\sin ^{-1} \tanh t
$$

In a final step, in order to fit $v$ in the interval $[-1 ; 1]$, we set $t=\left(t_{1}+t_{2}\right) / 2+v\left(t_{2}-t_{1}\right) / 2$. Then,

$$
\theta=\sin ^{-1} \tanh \left(\frac{t_{1}+t_{2}}{2}+\frac{v\left(t_{2}-t_{1}\right)}{2}\right) \quad \text { with } \mathrm{d} v=\frac{2 \mathrm{~d} \theta}{\left(t_{2}-t_{1}\right) \cos \theta}
$$

We have finally

$$
\begin{aligned}
& I=\iint_{T} \frac{r_{0}}{4}(1+u)\left(t_{2}-t_{1}\right) g(x(r(u, \theta(v)), \theta(v)), y(r(u, \theta(v)), \theta(v))) r(u, \theta(v)) \mathrm{d} u \mathrm{~d} v \\
& \quad \text { with }(u, v) \in[-1,1]^{2}
\end{aligned}
$$

As a result, we have a polynomial part in $u$, and a smooth part in $v$. Both are easily integrated with few sampling points. As one can see from Figure 9, the convergence has been highly improved for the five functions on which the integrand is based. The results have been obtained using the same number of Gauss points for each axis $(u$ and $v)$. The mapping is shown to be useful to integrate singular functions. We studied mainly the radial influence, since the angular functions are smooth, and thus, are less error prone. However, if the triangle is almost flat, it will have greater variations, so, more integration points will be required. This is not the case if the triangle is narrow. We plan to investigate this issue. As for $3 \mathrm{D}$ integration, the proposed mapping has to be adapted to the more complex case of tetrahedrons and eventually prisms. This will be presented upon completion in a subsequent paper.

Figure 9 clearly shows significant improvements in the convergence characteristics of the integration scheme with the singular mapping. Now one might wonder how to determine the number of integration points. Basically, we need at least 2 more significant digits in the coefficients of the stiffness matrix than the number of digits 'cancelled' because of the relatively bad condition number. In our case it would mean approximately 9 digits (with preconditioning). This leads to approximately 30 evaluations in singular elements. This means that when going further, one will not see much change in the coefficients obtained when solving the system. But convergence properties are not related directly to the fact that the weak form is integrated properly. The fact that under-integrated elements still lead to optimal convergence in regular F.E. is showing it. The important point is that there must be a sufficient number of sampling points to discriminate the different shape functions involved and obtain a non-singular matrix. 


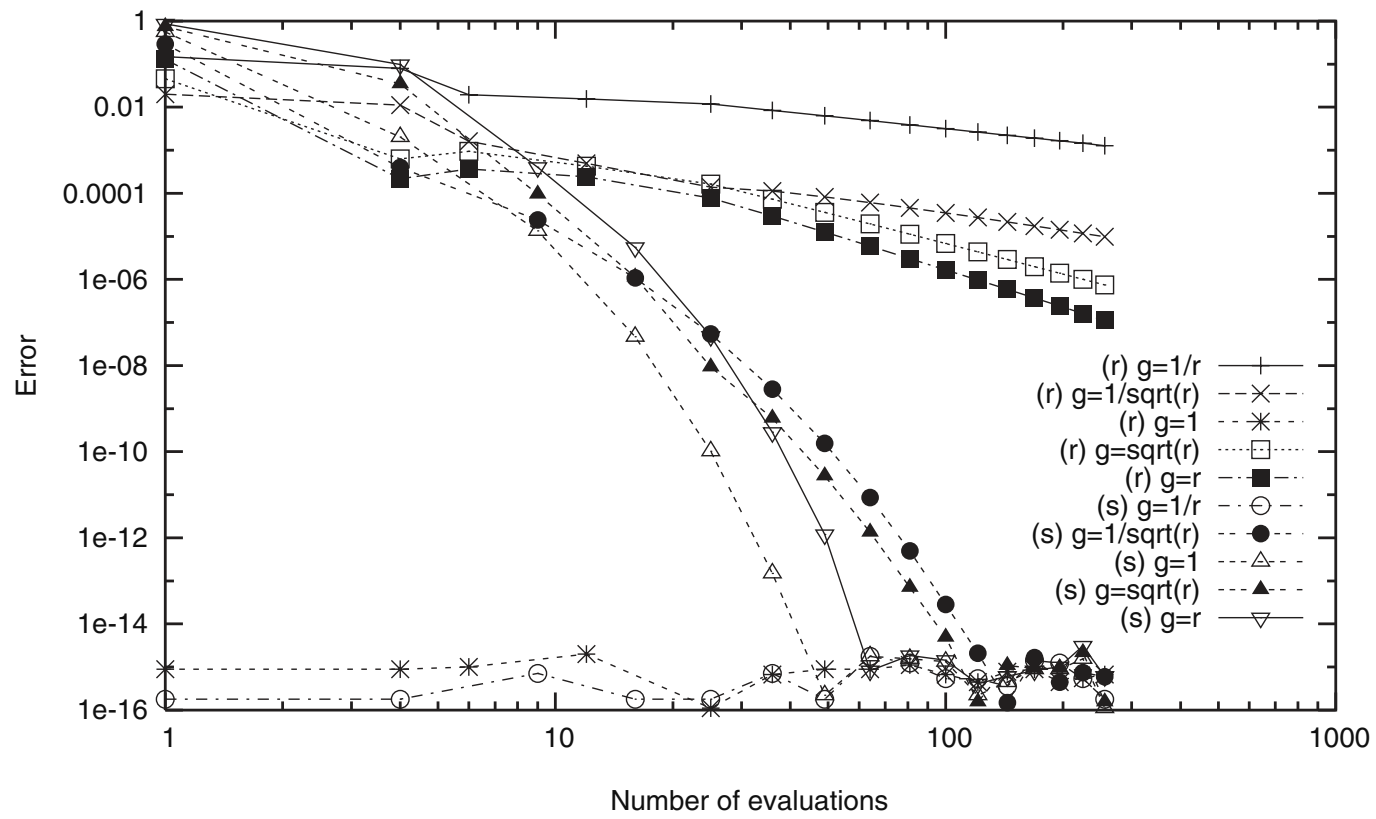

Figure 9. Convergence for a singular mapping (s) versus regular Gauss integration (r).

Under this assumption, the quadrature is drastically reduced to about 4 or 9 integration points. And then, an improved quadrature only permits to relate more easily the actual shape functions (as seen through the quadrature) and the real shape functions (as coded). In our case we actually did compute convergence curves with a regular high order Gauss quadrature (not shown here for the sake of brevity). These curves share the order of convergences with curves made with the new quadrature and presented in the sequel (see Section 5). This confirms that exact integration is not necessary for proper convergence.

The mapping described here is only valid 'as is' on the elements sharing one vertex with the crack tip. In fact, in the enriched area, there are elements which have a singular functional space, but have no vertex linked to the crack tip. It is desirable to integrate those elements with the same accuracy as displayed in Figure 9. It does not seem to be easy to set up an adapted mapping for that kind of problem, because of the rather high number of parameters involved. There is even no proof than one can find such a mapping in the general case. We have developed another approach, based on the superposition of up to three singular mappings to solve this problem. This uses the superposition of the accurate integrals obtained by linking successive edges of the triangle with the singular point of the enriched function. In Figure 10, we have the triangle 0 (solid lines) which is the one we want to integrate on. We know that we can integrate very accurately on triangles where one node lies on the crack front. So we decide to create up to three virtual triangles. These triangles are generated consecutively with two nodes of the original triangle and the node corresponding to the crack tip. The terms calculated on the artificial triangles are added, if the artificial and the original triangles have a non-empty intersection, or subtracted, if the intersection is empty. Finally, in the case of triangles close to (but not touching) the crack front, with the superposition technique, we 


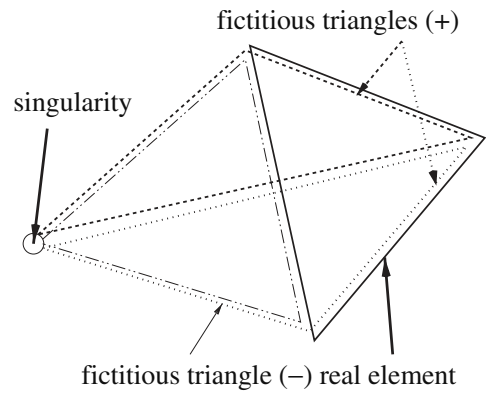

Figure 10. Decomposition of a 'real' triangle into three fictitious triangles.

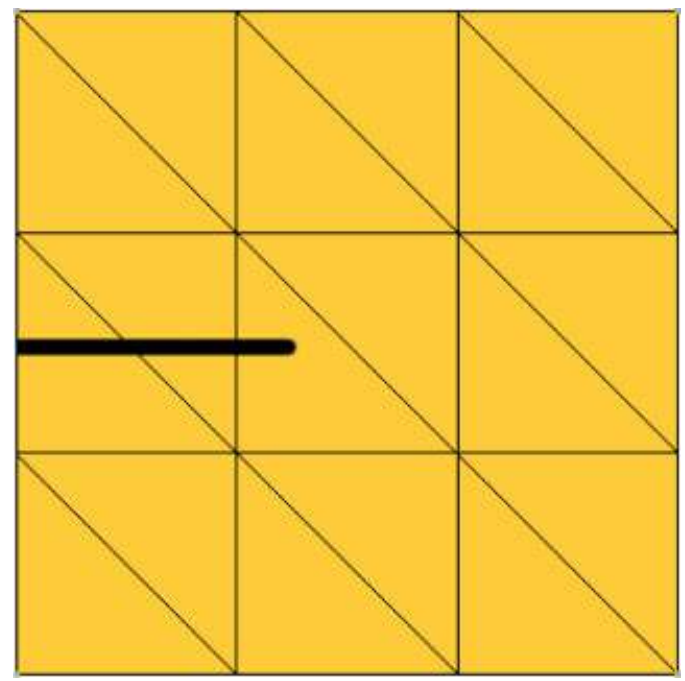

Figure 11. Integration benchmark on a simple mesh.

obtain the same convergence as in the case of a simple singular mapping with the crack front on a node. The drawback of this procedure is that for the same accuracy, there are three times as many evaluation points to consider. Also, this might lead to inaccuracies if the element is far from the crack front as values to be withdrawn from each others might be very similar. However, we wish to point out that for engineering problems, the geometrical enrichment domain will generally not be so wide and thus the superposition technique still works with a decent accuracy. We did not study thoroughly the limits of the algorithm but it is robust for our application (and one need to bear in mind that in the convergence study, for the most refined case, the geometrical enrichment was no more than 20 elements wide). One may add an additional remark concerning the non-linear case: the extension of non-linear variables might also be an issue, but then probably the existence of an asymptotic expansion available as enrichment function might be discussed.

This integration scheme is applied on a $3 \times 3$ regular mesh. The crack tip is located in the middle of an element (see Figure 11) and we study the convergence of the norm of the stiffness matrix. The plot is made considering the number of evaluations made on shape functions on 


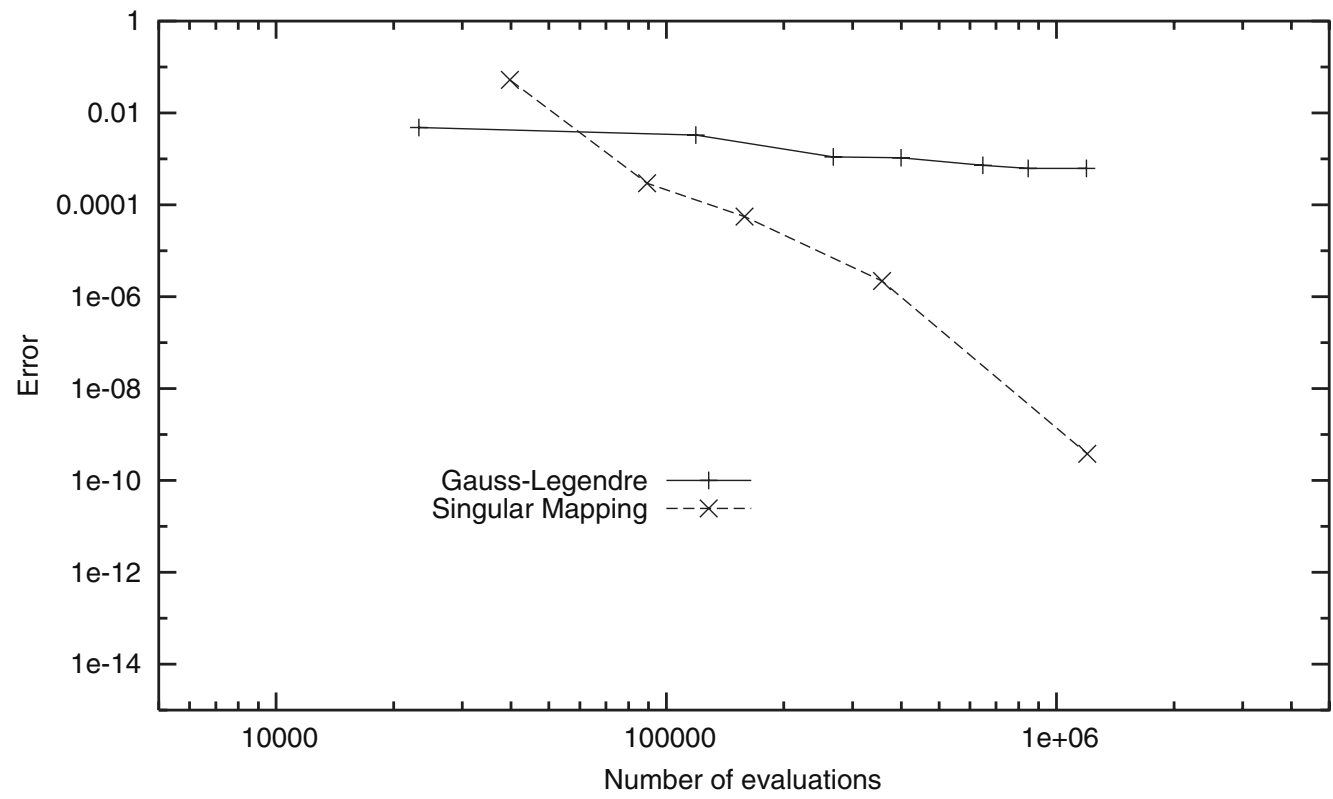

Figure 12. Convergence of the stiffness matrix norm for a singular mapping and a regular Gauss integration.

the $x$-axis, this includes the effect of splitting triangles and using fictitious triangles in the case of the singular mapping. The results in Figure 12 show an increased convergence with the new decomposition. The number of evaluations seem very high in this study, because high order integration schemes are used on elements which may have been split into up to 9 subtriangles. In addition, some elements contain up to 15 shape functions, leading to 120 'combinations' of shape functions to integrate. Altogether, for the whole domain, the number of evaluations may rise up to one million or more. As a comparison, for standard finite elements (no asymptotic enrichment), one would have 'only' around 800 shape function evaluation points for an exact integration, for the same problem. Note that the benchmark presented here is not representative of a real computation, it rather tells what happens at the crack tip. In real computations, most of the cpu time is spent solving the linear system; integration accounts only for a small percentage of the cpu time. In addition, the enriched domain is usually very small compared to the whole domain.

\subsection{Preconditioning}

The above-mentioned geometrical enrichment procedure produces ill-conditioned mass and stiffness matrices since many degrees of freedom (dof) are enriched. The use of iterative solvers, even with 'out of the box' built in preconditioners (incomplete LU among others) is difficult, especially in $3 \mathrm{D}$ when the number of degrees of freedom is high, as in mechanical models that are typically of interest to industry. We propose a specialized preconditioner for enriched finite elements. This preconditioner is not a substitute for general purpose preconditioners found in iterative solvers; its aim is to take advantage of the knowledge of the enrichment to produce 
linear systems that are easier to solve with 'off the shelf' preconditioners and solvers. The idea behind this specific preconditioning scheme is to orthogonalize the finite element basis generated for each regular degree of freedom. Let us consider the structure of the linear system $\mathbf{K u}=\mathbf{f}$

$$
\left[\begin{array}{llll}
\ddots & \vdots & \vdots & \\
& a & b & \cdots \\
& b & c & \cdots \\
& & & \ddots
\end{array}\right] \mathbf{u}=\mathbf{f}
$$

In the case displayed here, there is only one enrichment at a time, for the sake of clarity. Thus, in addition to each regular dof, there is one enriched dof. This gives the size (here, 2) of the sub-matrix showing terms $a, b$ and $c$. We want the regular and the enriched functions to be orthogonal in the $\mathbf{K}$ matrix (i.e. we want $b$ to be zero), in order to solve the modified linear system

$$
\left[\begin{array}{llll}
\ddots & \vdots & \vdots & \\
& a & 0 & \cdots \\
& 0 & c & \cdots \\
& & & \ddots
\end{array}\right] \tilde{\mathbf{u}}=\tilde{\mathbf{f}}
$$

To obtain this result, we do a Cholesky decomposition (Equation (29)) of the sub-matrix, and use this decomposition to pre- and post-multiply the appropriate terms in the relation. The matrix is completed with diagonal 'ones' to match the size of the original system. Of course the implementation is not exactly as described for obvious performance issues, but it is mathematically equivalent. Let $\mathbf{A}$ be the submatrix to orthogonalize

$$
\mathbf{A}=\left[\begin{array}{ll}
a & b \\
b & c
\end{array}\right]
$$

Let $\mathbf{G}$ be the lower triangular Cholesky decomposition of matrix $\mathbf{A}$

$$
\mathbf{A}=\mathbf{G G}^{\mathrm{T}}
$$

We take the inverse of the decomposition and scale it so that we keep the diagonal terms of the matrix $\mathbf{A}$ after the preconditioning. This also allows us to work only on the subset of the enriched dofs and keep the structure of the classical dofs. The idea of scaling is to avoid diagonal terms with very different magnitudes (at least not worse than with regular finite elements). Also, note that diagonal preconditioners are generally available in off-the-shelf iterative solvers.

Let $\mathbf{D}$ be the diagonal matrix made of the square root of diagonal values of matrix $\mathbf{A}$

$$
\mathbf{D}_{i j}=\sqrt{\mathbf{A}_{i j} \delta_{i j}} \quad \text { (no summation) }
$$




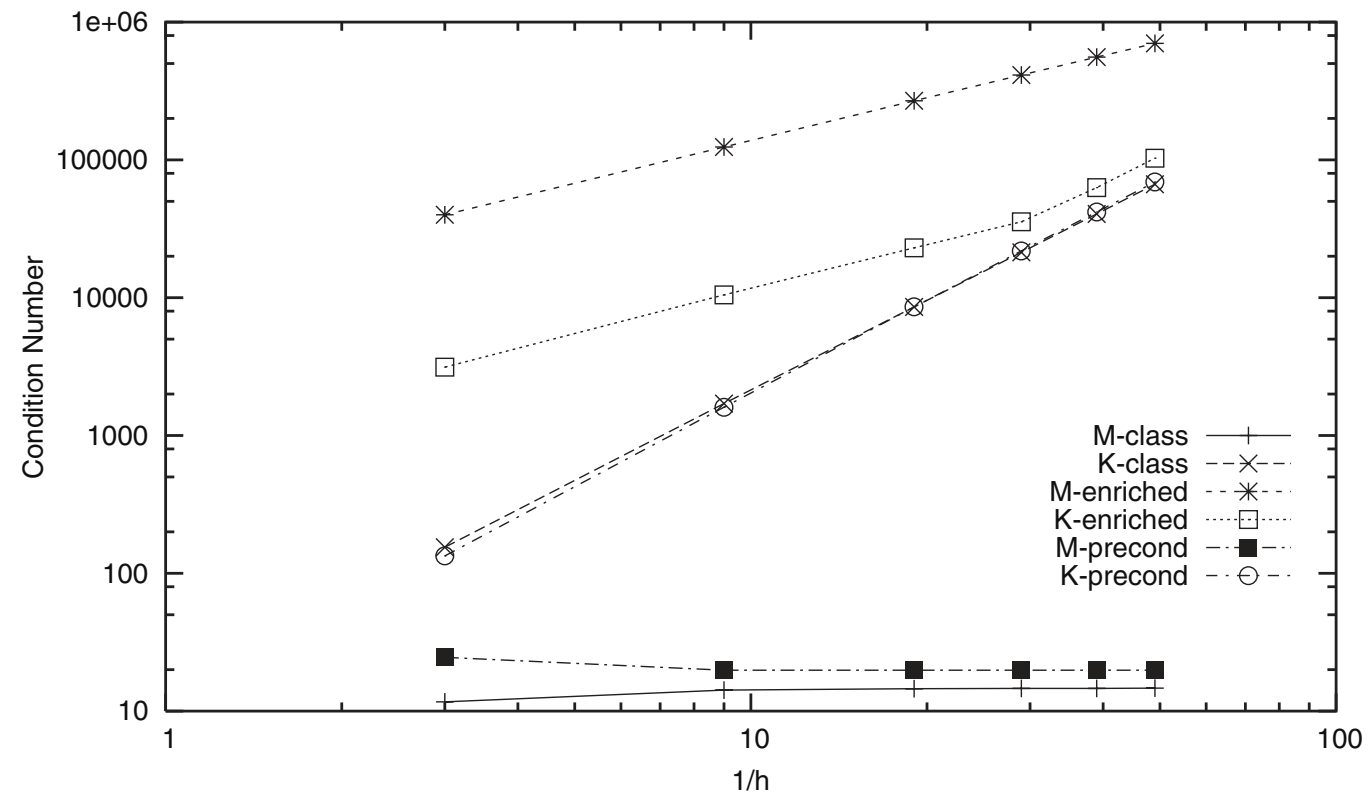

Figure 13. Preconditioned versus raw condition number for enriched $\mathbf{K}$ and $\mathbf{M}$ matrices (topological enrichment). For reference the condition numbers for unenriched $\mathbf{K}$ and $\mathbf{M}$ matrices are also indicated.

We have then

$$
\mathbf{R}=\mathbf{G}^{-1} \mathbf{D}
$$

This is the preconditioning submatrix for only one set of enriched dofs. The full preconditioner matrix $\mathbf{R}^{*}$ is then built and augmented with every submatrix $\mathbf{R}$ obtained for each group of enriched dofs. The resulting system is then

$$
\mathbf{R}^{*} \mathbf{K R}^{* \mathrm{~T}} \tilde{\mathbf{u}}=\mathbf{R}^{*} \mathbf{f} \quad \text { with } \mathbf{u}=\mathbf{R}^{* \mathrm{~T}} \tilde{\mathbf{u}}
$$

If needed, one can eventually skip the scaling with matrix $\mathbf{D}$ and directly use $\mathbf{R}=\mathbf{G}^{-1}$ as the preconditioning matrix. This will lead to a system with a diagonal consisting only of ones. This way, the preconditioner also acts as a diagonal preconditioner. This is not the objective here as we want to apply the preconditioning scheme only on enriched nodes or dofs, so we leave Equation (31) unmodified.

The procedure is tested on a unit 2D square patch, with the enrichment (geometrical and topological) as described above. We examine both mass and stiffness matrices since both are widely used in mechanical simulations. Note that for an implicit Newmark scheme in dynamic simulations, one should precondition a linear combination of the stiffness and mass matrices. As a comparison, it should be mentioned that for linear finite elements without enrichment, the condition number of mass matrices tends to a constant with increasing refinement, whereas that of stiffness matrices increases like $1 / h^{2}$. With enrichment, and especially with geometrical 


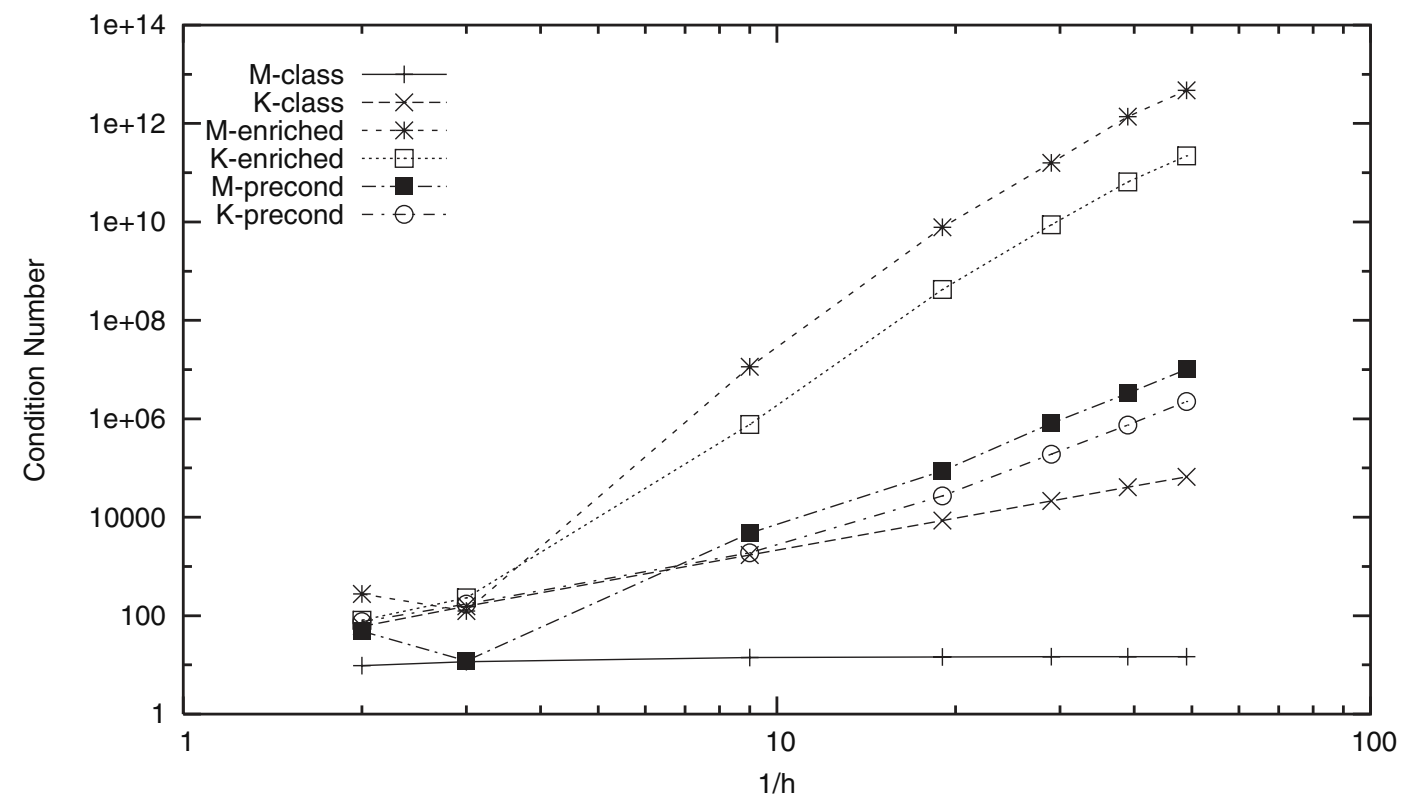

Figure 14. Preconditioned versus raw condition number for $\mathbf{K}$ and $\mathbf{M}$ matrices (geometrical enrichment). For reference the condition numbers for unenriched $\mathbf{K}$ and $\mathbf{M}$ matrices are also indicated.

enrichment, the condition number degrades quickly as the mesh density $d=1 / h$ increases. Figures 13 and 14 show the results obtained by the preconditioner.

In the case of the mass matrix $\mathrm{M}$, the condition number is greatly reduced for both topological and geometrical enrichments using the new strategy. It is however still increasing with the mesh density in the case of geometrical enrichment. For the stiffness matrix $\mathrm{K}$, the results are globally the same. In the case of topological enrichment, one can see that the condition number for non-preconditioned matrices is very close to the curve corresponding to regular finite elements. However, we observed that preconditioning allows one to solve large systems that were otherwise very difficult to solve using iterative solvers. When the enrichment is geometrical, even for moderately sized problems, standard iterative methods fail without that ad hoc preconditioner. It should be mentioned that the above preconditioning scheme could be used for any type of enrichment, and is local with respect to the dofs. In particular, it could be used in conjunction with the X-FEM material interface enrichment scheme [16].

\section{CONVERGENCE RESULTS}

In this section we shall describe current results obtained using (a) a regular finite element procedure without enrichment and a meshed crack, (b) results obtained by the X-FEM method with topological enrichment and (c) results obtained by a geometrical enrichment. We consider the domain $\Omega=[0,1]^{2}$ under tension. The tension corresponds to an infinite cracked body uniformly loaded such as $K_{1}=1$. The exact stress field applied is well known from the 

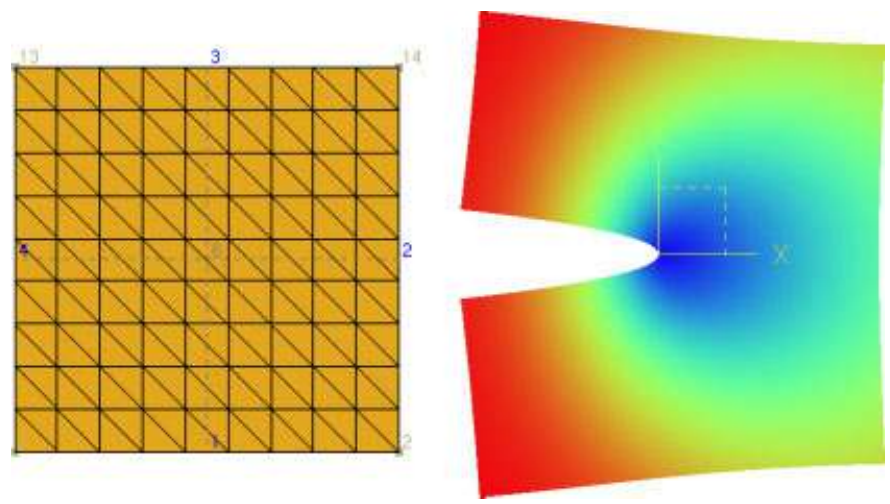

Figure 15. 2D domain mesh (left) with exaggerated displacement field (right) corresponding to a far loading such that $K_{1}=1$.

classical Westergaard solution. The load $\mathbf{t}$ (34) is applied on all four sides of the patch where $\mathbf{n}$ is the exterior normal to the domain. The rigid body modes are removed by three scalar appropriate Dirichlet boundary conditions.

$$
\begin{aligned}
\sigma_{11} & =\frac{1}{\sqrt{2 \pi r}} \cos \frac{\theta}{2}\left(1-\sin \frac{\theta}{2} \sin \frac{3 \theta}{2}\right) \\
\sigma_{12} & =\sigma_{21}=\frac{1}{\sqrt{2 \pi r}} \cos \frac{\theta}{2} \sin \frac{\theta}{2} \cos \frac{3 \theta}{2} \\
\sigma_{22} & =\frac{1}{\sqrt{2 \pi r}} \cos \frac{\theta}{2}\left(1+\sin \frac{\theta}{2} \sin \frac{3 \theta}{2}\right) \\
\mathbf{t} & =\sigma \cdot \mathbf{n}
\end{aligned}
$$

Figure 15 (left) shows the structure and (right) the exact displacement field. As one can see, the crack geometry is not aligned with the mesh. In the case of geometrical enrichment, the enrichment is made over a circular patch with $r_{e}=0.05$. In this case, nodes inside this circular patch (for which $r \leqslant r_{e}$ ) are enriched. In the case of a topological enrichment, it is made on the elements immediately around the crack tip (one layer of elements). The nodes belonging to elements immediately around the crack tip are enriched.

\subsection{Error in the energy}

The convergence results in relation to the element size $h$ show an improvement as one chooses to enrich in a geometrical way instead of a topological way. In linear finite elements, when the exact displacement field is smooth, the theoretical rate of convergence in the energy norm is $O(h)$ for linear elements. We wish to clarify the vocabulary used in the sequel. What we mean by optimal convergence rate is for linear finite elements for smooth problems. In their seminal paper Babuška et al. [2], also define it this way. When the solution is not smooth, the rate of convergence is less than unity. For a crack, it is $O\left(h^{1 / 2}\right)$. This appears on Figure 16, 


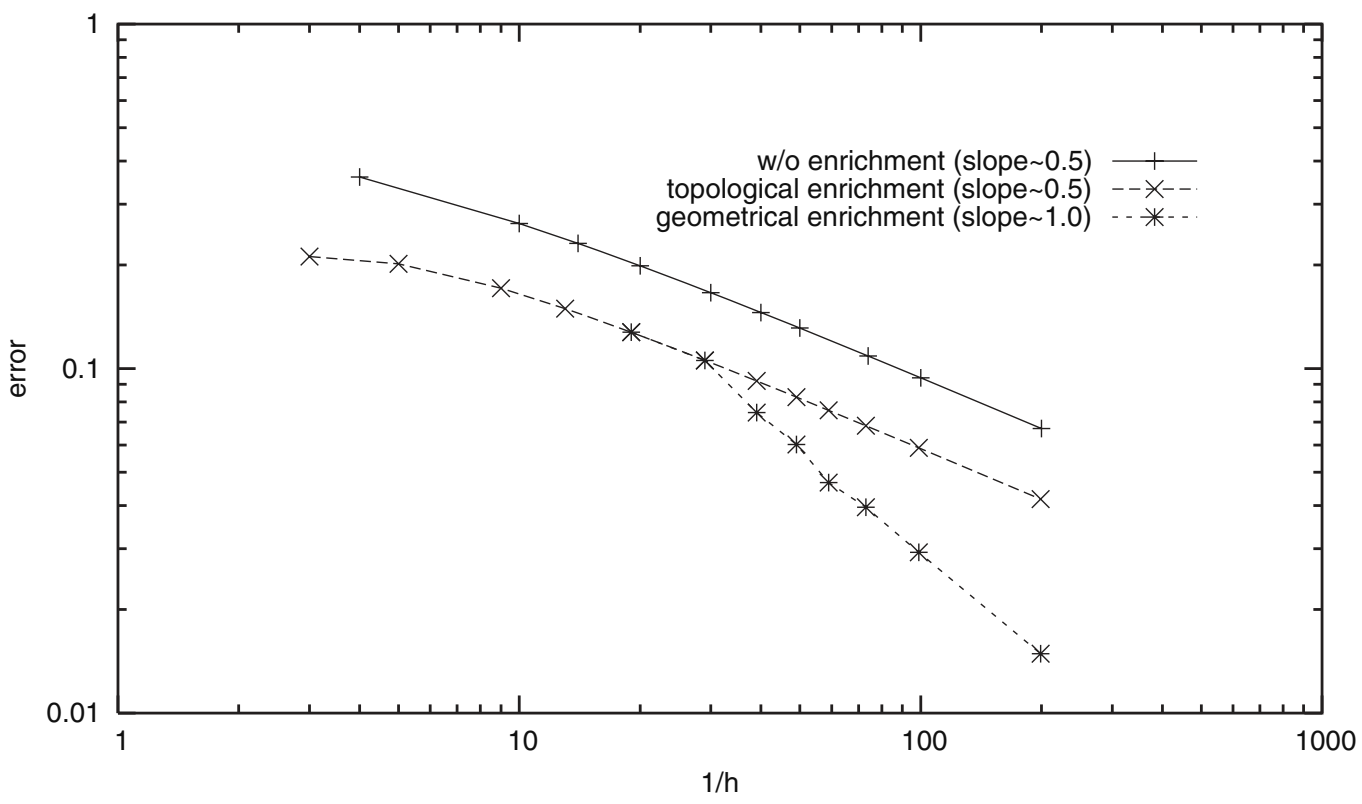

Figure 16. Energy norm error for three enrichment schemes $(1 / h$ scale).

which shows convergence results for unenriched as well as enriched displacement fields. When one chooses to enrich the displacement field around the crack tip in a topological way, the graph shows an almost constant shift toward lower error when compared to unenriched finite elements. This implies that the enrichment procedure does not improve the convergence rate as one could expect by the addition of exact displacement modes in the enriched field. Only the constant in the log-log convergence curve is improved, not its slope. When one chooses to enrich in a geometrical way, the optimal rate of convergence (again, for linear finite element and a smooth solution) is achieved. The actual convergence rate observed toward the right of the diagram lies between 0.95 and 1.05. In this calculation, the exact strain was computed on the exact displacement field (known). The part of the graph below $1 / h=20$ needs further explanation. We did not provide results for the geometrical enrichment because when $1 / h \leqslant 20$ (note that the radius we have chosen for the enrichment is 0.05 ), it is not guaranteed to include any element, leaving only the modified Heaviside enrichment. The crack tip is therefore not well located (roughly one element ahead of the desired crack tip position). In fact, in this case, we will of course choose to enrich at least the elements containing the crack tip. The second plot on Figure 17 is made relative to the number of dofs $N$. It is a better measure than $1 / h$, because it is directly related with the computational time. Again, the geometrical enrichment achieves a convergence rate close to optimal (in 2D, it is 1.0 also if one takes $\sqrt{N}$ as the $x$-axis, as here). A quick analysis shows that the convergence rate $\beta_{\sqrt{N}}$ achieved in a $\sqrt{N}$ scale is related to the ratio $\eta$ between the number of enriched dofs and non-enriched dofs

$$
\frac{\beta_{\sqrt{N}}}{\beta_{1 / h}}=\frac{1}{\sqrt{1+4 \eta}}
$$




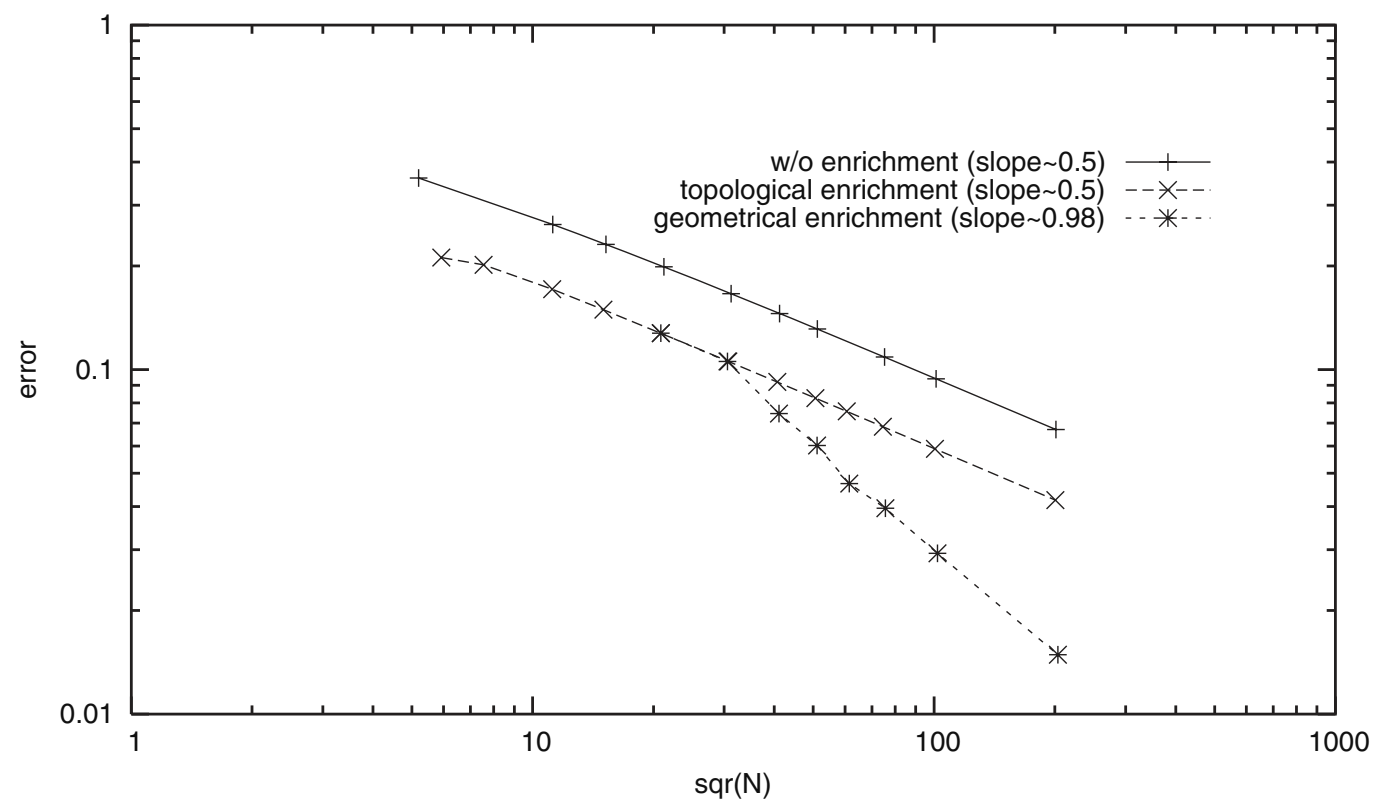

Figure 17. Energy norm error for three enrichment schemes $(\sqrt{N}$ scale).

Accordingly, if one enriches all dofs, the convergence rate will drop to $1 / \sqrt{5} \approx 0.45$. This is slightly lower than using no enrichment at all. However, there is no reason why one should enrich the whole domain. In fact, the convergence rate quickly gets very close to 1.0 as the ratio $\eta$ decreases. In our case, with a geometrical area covered by the enrichment being relatively big compared to the size of the domain (a disc $\frac{1}{10}$ in diameter, with $\eta=\pi / 100$ ), the theoretical 'optimal' rate of convergence in function of $\sqrt{N}$ is 0.985 . The one actually achieved is 0.98 . As a matter of comparison, the theoretical convergence rate for quadratic triangular elements with a crack and without geometrical enrichment is $O\left(h^{1 / 2}\right)$, same as linear elements, and a mere order $\frac{1}{4}$ in function of $\sqrt{N}$. As shown here, for moderately sized enrichment domains, the convergence is still close to optimal. In the sequel, we do have the same domains with the same enrichment areas so we will only provide curves in function of $1 / h$, as convergence results are usually expressed in this form.

\subsection{Errors in SIF computations}

The above convergence study is repeated for $J$ and interaction integrals. However, there is one more parameter, namely the size of the $J$-integral domain. We have defined two strategies: a domain whose size decreases with $h$, and a domain whose size is constant. This is very similar to the enriched area definition, so we shall call them later on with the same qualifiers: topological if the domain size is proportional to $h$ and geometrical if it is constant. As a consequence, the study involves four cases. The problem studied here is the same as for the energy norm error and, by construction, the exact solution for this problem is such that $K_{1}=1$ and $J=1$. The $J$-integral domain is a circular patch. Its radius is defined as: $r_{i}=0.15$ if it 


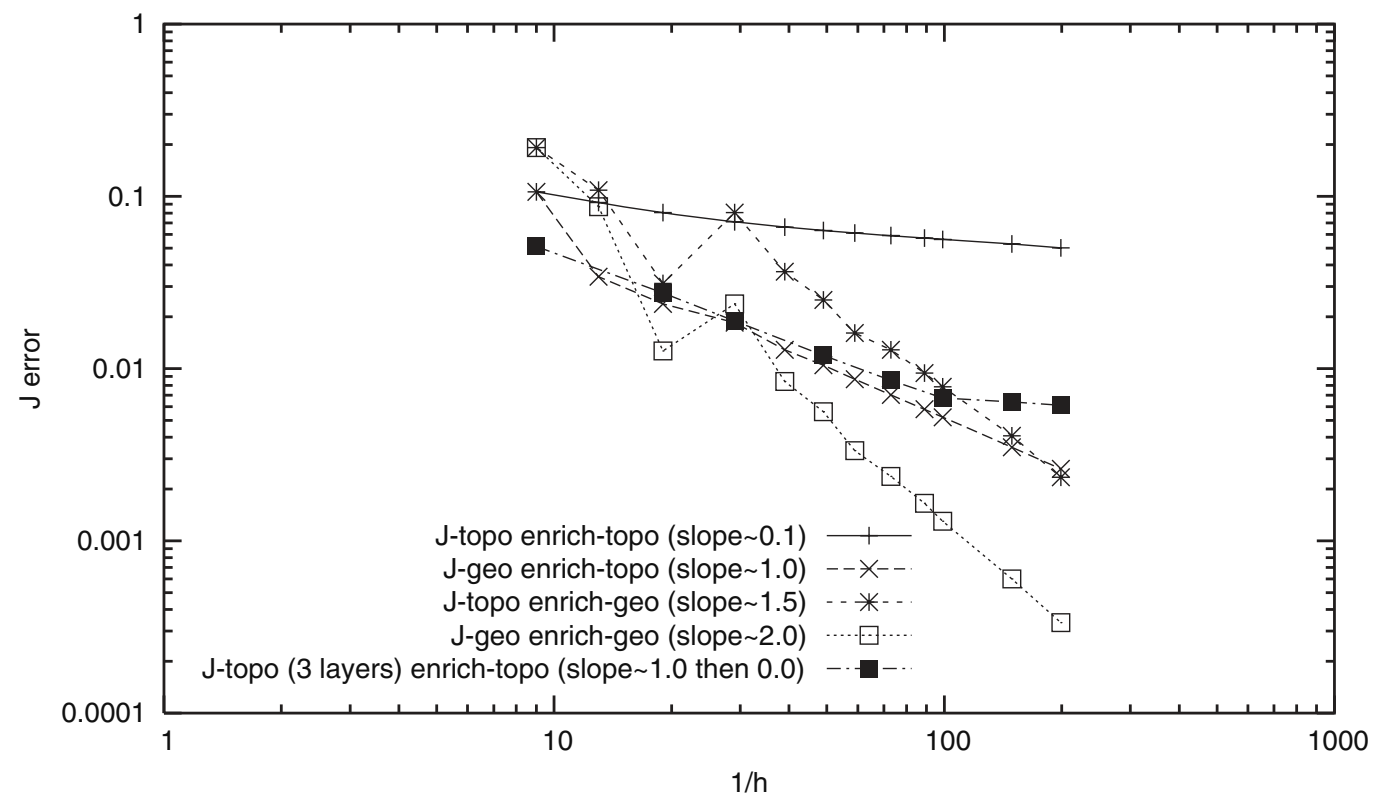

Figure 18. Error in $J$ versus element density.

is geometrical, and $r_{i}=1.5 h$ if it is topological (if $h=\frac{1}{10}$, both domains are the same). In addition, it should be noted that $J$ and $K_{1}$ have here a local meaning. The stress intensity factors $K$ are determined using the formulas of Section 3. The convergence results for $J$ and $K_{1}$ are shown in Figures 18 and 19. In Figure 18, it is remarkable that there is a very slow convergence for a topological enrichment and a topological $J$-integral domain (first curve). In fact, this is the reason why one always recommends increasing the $J$-integral domain's size until convergence. To illustrate this, the last curve on the same figure presents the same case where the $J$-integral domain is composed by 3 layers of elements instead of 2 . Down to a relatively low error, there is convergence, but below an error of 0.01 , there is also a plateau indicating poor convergence. The second curve; which displays the convergence for a topological enrichment and a geometrical $J$-integral domain shows a convergence rate around 1.0, which is quite good. In the case of a geometrical enrichment, results are much better. Even in the case of a topological $J$-integral domain, there is convergence (the rate is in this case between 1.0 and 1.5 , see 3rd curve for both $J$ and $K_{1}$ ). This means that we are able to extract local information from the displacement field, and this even at the crack tip, which is an interesting feature. When both the enrichment and the $J$-integral domain are geometrical, the convergence rate is increased to around 2.0 for both $J$ and $K_{1}$ and provides the best approximation. However, the very fast convergence of the $J$-integral with increasing number of layers (see curves 1 and 5 in Figure 18 for, respectively, 1 and 3 layers of elements in the domain integrals) makes no sense to use domain size beyond 5 or 6 layers of elements. At that point, the error in $J$ and $K_{1}$ is dominated by the approximation error in the displacement field. A comparison between Figures 18 and 19 shows that the error in $K$ decreases as the same order as the error in $J$. However, it also shows that the error in $K$ is about half that of $J$. This is obvious since $J \approx K^{2}$, so $\delta J / J \approx 2(\delta K / K)$. 


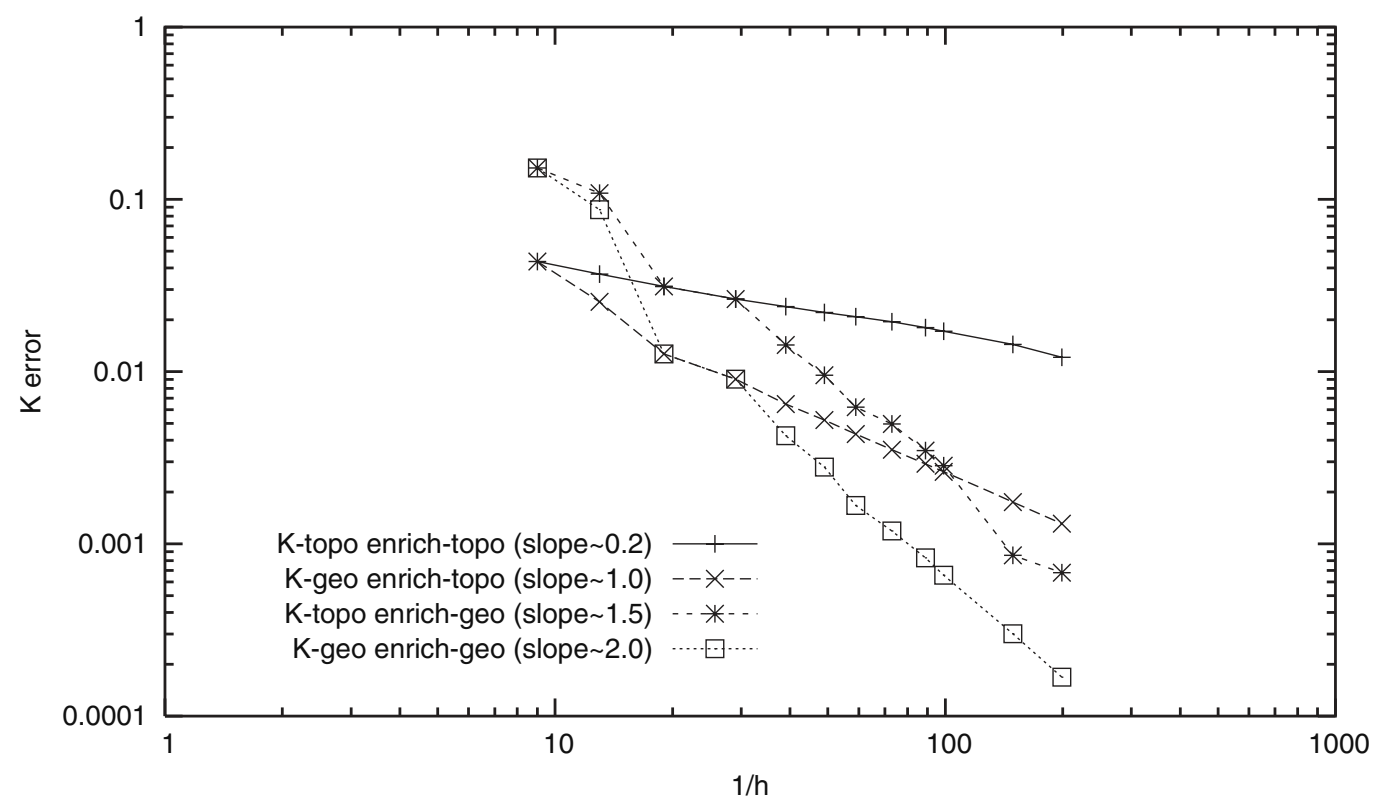

Figure 19. Error in $K_{1}$ versus element density.

\section{CRACK PROPAGATION EXAMPLES}

We present here two examples of crack propagation. In these examples, the crack propagates with respect to the Paris fatigue law. The numerical scheme for the computation (enrichment, SIFs calculation and preconditioning) is as presented earlier in the text, except for the integration made with a standard Gauss-Legendre rule (we do not have singular integration scheme yet available for arbitrary 3D meshes). We used a very high number of Gauss points for better accuracy, this makes the stiffness matrix assembly stage a very time consuming process. The level-set update scheme is not described here; it is similar to the previously published paper [17], with some enhancements in the propagation stage (after velocity extension and before reinitialization and re-orthogonalization). We provide those examples to show the reader that developments made in this paper are available for arbitrary 3D computations, except the integration of 3D singular function. These are preliminary results in $3 \mathrm{D}$ and hence we do not provide extensive comparisons with previously published material.

\subsection{Initially straight crack}

The first example in Figure 20 is an initially straight crack in with a combined shear-traction boundary condition. The traction is vertical, and the shear is applied on all four lateral sides. The crack must then suddenly follow a kinked path. Note that it is not an obvious task for the level-set updating algorithms; these are best for representing smooth interfaces. The domain is a unit cube and we use reduced material properties $(E=1$ and $v=0)$ to perform this simulation. 

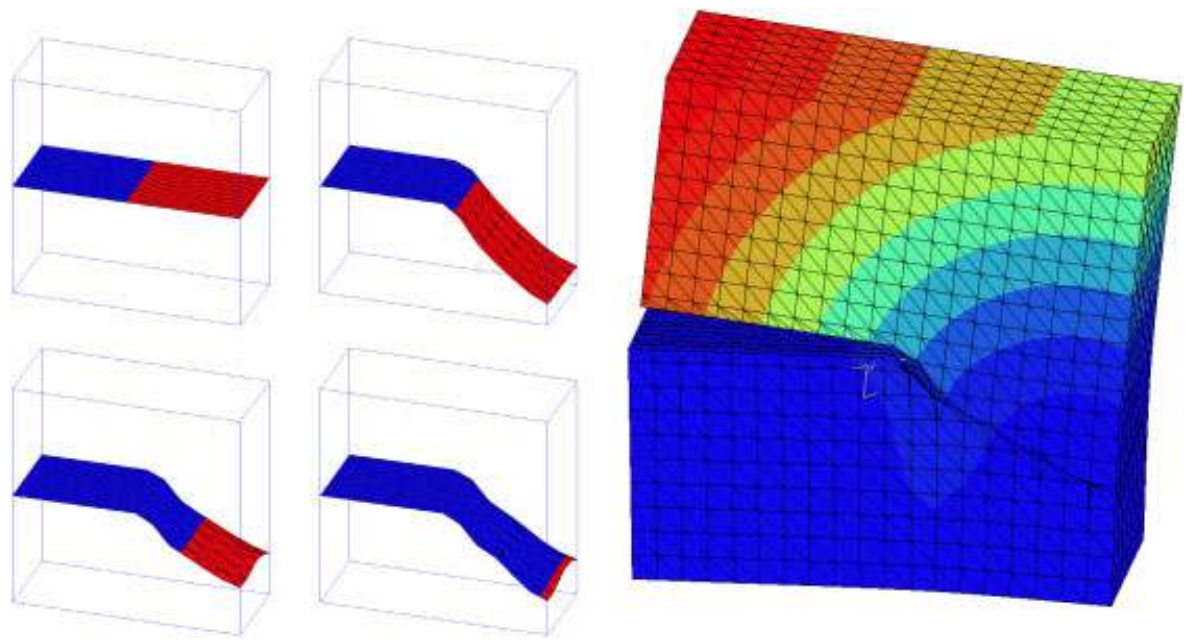

Figure 20. Initially straight crack: front positions (left), and displacement field after a few time steps (right). The loading is a combination of uniform (vertical) tension and shear.

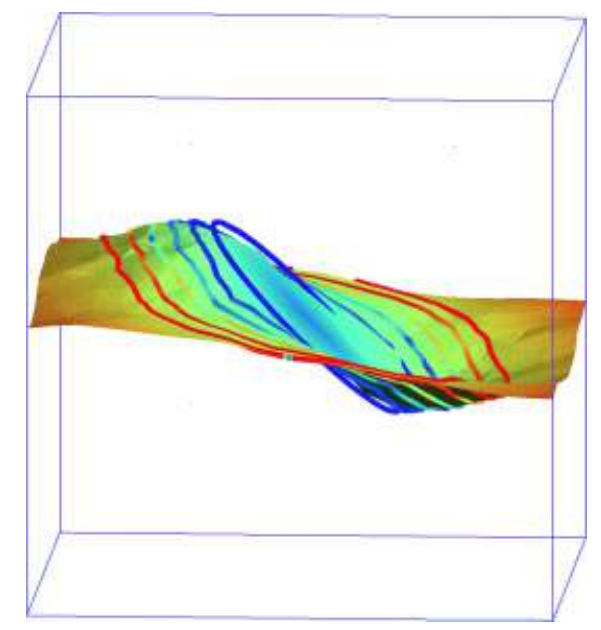

Figure 21. Penny-shaped crack: crack surface and successive crack front positions. The loading is uniform (vertical) tension.

\subsection{Penny-shaped crack}

The second example in Figure 21 is the problem of a inclined penny shaped crack under uniform vertical tension. Here, there is a change in the topology of the crack front. It is initially circular, and after several propagation steps, it will end up split into four subfront in each corner of the domain. This is where level sets have a definite advantage over other models 
for the crack geometry. The domain is a unit cube and we use reduced material properties $(E=1$ and $v=0)$ to perform this simulation.

\section{CONCLUSIONS AND FUTURE WORK}

We have improved the robustness of the X-FEM method by (a) introducing a different enrichment scheme; (b) developing new integration quadrature for asymptotic functions and (c) implementing a preconditioning scheme adapted to the enriched functions. The work is mainly focused on a 2D benchmark problem. The enrichment scheme presented in this paper achieves a better convergence rate. However, this has adverse effects on the conditioning properties of the resulting linear system. We addressed this issue by improving the integration and provided a preconditioning scheme. Future work is planned to study the 3D case. We plan also to work on an error estimator that would allow us to set the enrichment radius and the mesh refinement needed for a prescribed accuracy. This is necessary because we introduced the enrichment radius in the formulation, which is a new parameter. This new parameter may be determined by geometrical considerations (much alike the St Venant principle) but one has to bear in mind that it has no relation with the physics. For models showing a physical length scale, for instance cohesive cracks, there may be a relation between that length scale and an 'optimal' value for the new parameter. This is however not the topic of the present publication.

We bring to the attention of the reader another publication to be submitted [18] dealing with a similar topic. We were unaware of this paper at time of submission. This paper corroborate our results but also brings to light other convergence properties of the X-FEM.

\section{REFERENCES}

1. Barsoum RS. Application of quadratic isoparametric elements in linear fracture mechanics. International Journal of Fracture 1974; 10:603-605.

2. Babuška I, Melenk JM. The partition of unity method. International Journal for Numerical Methods in Engineering 1997; 40:727-758.

3. Strouboulis T, Babuška I, Copps K. The design and analysis of the generalized finite element method. Computer Methods in Applied Mechanics and Engineering 2000; 181:43-69.

4. Moës N, Dolbow J, Belytschko T. A finite element method for crack growth without remeshing. International Journal for Numerical Methods in Engineering 1999; 46:131-150.

5. Belytschko T, Black T. Elastic crack growth in finite elements with minimal remeshing. International Journal for Numerical Methods in Engineering 1999; 44:601-620.

6. Strouboulis T, Copps K, Babuška I. The generalized finite element method: an example of its implementation and illustration of its performance. International Journal for Numerical Methods in Engineering 2000; 47:1401-1417.

7. Berntson J, Espelid TO, Genz A. An adaptive algorithm for the approximate calculation of multiple integrals. ACM Transactions on Mathematical Software 1991; 17:437-451.

8. Destuynder P, Djaoua M, Lescure S. Some remarks on elastic fracture mechanics. Journal de Mécanique Théorique et Appliquée 1983; 2:113-135.

9. Shih CF, Moran B, Nakamura T. Energy release rate along a three-dimensional crack front in a thermally stressed body. International Journal of Fracture 1986; 30:79-102.

10. Rice JR. A path independent integral and the approximate analysis of strain concentrations by notches and cracks. Journal of Applied Mechanics 1968; 35:379-386.

11. Moës N, Gravouil A, Belytschko T. Non-planar 3D crack growth by the extended finite element and level sets-Part I: mechanical model. International Journal for Numerical Methods in Engineering 2002; 53: $2549-2568$. 
12. Gosz M, Moran B. An interaction energy integral method for computation of mixed-mode stress intensity factors along non-planar crack fronts in three dimensions. Engineering Fracture Mechanics 2002; 69: $299-319$.

13. Bui HD. Mécanique de la rupture fragile (in French). Masson: Paris, 1978.

14. Chessa J, Wang H, Belytschko T. On the construction of blending elements for local partition of unity enriched finite elements. International Journal for Numerical Methods in Engineering 2003; 57:1015-1038.

15. Burgardt $\mathrm{B}$. Contribution à l'étude des méthodes des équations intégrales et à leur mise en oeuvre numérique en élastoplasticité (in French). Thèse de doctorat, Université de Nantes, 1999.

16. Moës N, Cloirec M, Cartraud P, Remacle J-F. A computational approach to handle complex microstructure geometries. Computer Methods in Applied Mechanics and Engineering 2003; 192:3163-3177.

17. Gravouil A, Moës N, Belytschko T. Non-planar 3D crack growth by the extended finite element and level sets-Part II: level set update. International Journal for Numerical Methods in Engineering 2002; 53: 2569-2586.

18. Laborde P, Pommier J, Renard Y, Salaün M. High order extended finite element method for cracked domains. International Journal for Numerical Methods in Engineering 2004, submitted.

19. Béchet E, Minnebo H, Moës N, Burgardt B. Convergence and conditioning issues with X-FEM in fracture mechanics. WCCM6, 5-10 September 2004, Beijing. 